\title{
A Case of Recurrence/Refractory Mediastinal Hodgkin's Lymphoma Relapsed After Allogeneic Hematopoietic Stem Cell Transplantation: Immunotherapy Brings Hope
}

\author{
Jiaxin Wang, Jiaxiong Tan, Yuhong Lu* \\ Department of Hematology, First Affiliated Hospital, Jinan University, Guangzhou, China \\ Email address: \\ merryveraforever@163.com (Jiaxin Wang),gdydtjx@163.com (Jiaxiong Tan),yhl2006jn@163.com (Yuhong Lu) \\ ${ }^{*}$ Corresponding author
}

To cite this article:

Jiaxin Wang, Jiaxiong Tan, Yuhong Lu. A case of Recurrence/Refractory Mediastinal Hodgkin's Lymphoma Relapsed After Allogeneic Hematopoietic Stem Cell Transplantation: Immunotherapy Brings Hope. International Journal of Immunology. Vol. 9, No. 3, 2021 , pp. 54-58. doi: $10.11648 /$ j.iji.20210903.13

Received: September 3, 2021; Accepted: September 17, 2021; Published: September 26, 2021

\begin{abstract}
Hodgkin lymphoma (HL), is a B-cell lymphoma. HL is a common hematological malignancy, mostly seen in young and middle-aged people. According to NCCN guidelines, common treatment options include ABVD, BEACOPP, StandfordV, etc. Most HL have a good prognosis and even can be cured. However, some Refractory / Recurrent (R/R) HL, continue to progress during treatment or even relapse after the completion of a complete chemotherapy course 3 months. At this time, the best treatment for R/R HL is high-dose chemotherapy / Autologous Hematopoietic Stem Cell Transplantation (HDC/HSCT). This paper reports a case of Recurrence/Refractory $(R / R)$ mediastinal Hodgkin's lymphoma that relapsed after Allogeneic Hematopoietic Stem Cell Transplantation. After receiving a variety of treatments, including chemotherapy, radiotherapy, autologous hematopoietic stem cell transplantation and so on, no long-term sustained remission was achieved. Traditional chemotherapy regimen has not brought ideal therapeutic effect to patients, but the rapid development of immunotherapy in recent years has brought new hope to patients. Finally sustained remission was achieved under the treatment of Brentuximab Vedotin combined with PD-1 inhibitor. This paper combined with the literature reports and discussed the clinical characteristics, treatment process and related experience and thinking methods of immunotherapy related to PD-1 inhibitors in this refractory and easy-to-relapse case. To provide more clinical data for the application of immunotherapy in R/R HL.
\end{abstract}

Keywords: Hodgkin Lymphoma, Refractory / Recurrent, Brentuximab Vedotin, PD-1 Inhibito, Immune

\section{Introduction}

Hodgkin lymphoma (HL), is a B-cell lymphoma. In the world, HL accounts for $13.6 \%$ of lymphoma. Most HL have a good prognosis and even can be cured [1]. However, some refractory / recurrent $(\mathrm{R} / \mathrm{R}) \mathrm{HL}$, continue to progress during treatment or even relapse after the completion of a complete chemotherapy course 3 months [2]. At this time, the best treatment for $\mathrm{R} / \mathrm{R} \mathrm{HL}$ is high-dose chemotherapy / Autologous Hematopoietic Stem Cell Transplantation (HDC/HSCT) [3]. This paper reports a case of R/R HL that relapsed three months after Allogeneic Hematopoietic Stem Cell Transplantation (Allo-HSCT). After diagnosis, the patient used BEACOPP for 8 cycles, but completing the chemotherapy, the lymphoma relapsed on the 43 rd day. Then came to hospital for Allo-HSCT, after acquired remission by using IGDV+ Brentuximab Vedotin (BV) chemotherapy regimens. However, it relapsed again in the second month after transplantation. At this time, there was no long-term sustained remission after using a variety of treatment regimens, including radiotherapy, chemotherapy and demethylation therapy. Finally, long-term sustained remission was achieved under the treatment of BV combined with Procedural death factors 1 (PD-1) inhibitors. This paper combined with the literature reports and discussed the clinical characteristics, treatment process and related experience and thinking methods of immunotherapy related to PD-1 inhibitors in this refractory and easy-to-relapse case. To provide more clinical data for the application of immunotherapy in R/R HL. 


\section{Case Report}

An 18-year-old man was treated for "repeated cough and expectoration (duration not mentioned)", coughing a small amount of yellow sticky sputum, with chest tightness, skin itching, no systemic symptoms (fever, weight loss, night sweats). PET/CT showed multiple enlarged lymph nodes with increased metabolic activity in bilateral supraclavicular fossa, mediastinum and left internal breast area, and abnormal metabolic activity without observed in the other parts. Then mediastinal lymph nodes biopsy was performed, immunohistochemistry showed: $\mathrm{LCA}(+), \quad \mathrm{CD} 30(+)$, CD15(+,partly), MUM1(+), CD3(-), CD5(-), CD7(-); ALK(-), EMA(-), PAX-5(+weakly), Oct-2(-), CD20(-), CD57(-), Granzyme--B(-), CD21(+, Follicular dendritic network).

The bone marrow examination without showed abnormality, the patient was diagnosed as HL mixed cell type, stage II A type. Within 16 months after definite diagnosis, remission was achieved after 8 courses of treatment with BEACOPP regimen. Forty-three days later, the patient was hospitalized again because of cough, expectoration and fever. PET/CT showed recurrence of lymphoma with invasion of both lungs. Right mediastinal lymph node and right upper lung nodule biopsy: IHC: LCA(-), CD20(-)CD30(+), CD15(+,partly), MUM-1(+), CD10(-), ALK(-), Bcl-6(-), CD23(-), CD5(-), Pax8(-). After 3 courses of IGDV (Ifosfamide $3400 \mathrm{mg} \mathrm{d} 1-2,3450 \mathrm{mg}$ d3-4+Gemcitabine $1400 \mathrm{mg} \mathrm{d} 1,1500 \mathrm{mg} \mathrm{d} 4+$ Vinorelbine 37 mg d1+methylprednisolone $100 \mathrm{mg} \mathrm{d} 1-4+$ Mesna $800 \mathrm{mg}$ tid) Hodgkin regimen and 4 cycles of BV (100 mg), PET/CT showed that the lesions were significantly reduced and partially disappeared.

Then the patient came to our hospital for further treatment, re-evaluated the patient's condition, and diagnosed as HL (mixed cell HL, typical stage IV, group B, IPI score 3, ECOG $0)$. The primary disease recurred during regular chemotherapy, had the indicator of Allo-HSCT. The donor is his father, HLA match type 5/10, blood type A, after pretreatment (Busulfan $12 \mathrm{mg} / \mathrm{Kg}+$ fludarabine + Cyclophosphamide), Allo-HSCT was performed. Cyclosporine A (CsA) + Mycophenolate Mofetil (MMF) + high dose Cyclophosphamide (CY) was used to resist graft versus host disease (GVHD). The lymphoma relapsed again in the third month after transplantation. And then local radiotherapy was given to the primary focus for 18 times ( 2 gy/times). After radiotherapy, chest CT showed the mediastinal and right hilar masses of were enlarged, and the infiltrative foci of lymphoma in both lungs were smaller than before.

Two months after radiotherapy, the patient had cough, expectoration, and fever once more. The re-examination of chest CT showed that the size of the primary mass was the same as before, while the nodules in both lungs increased and pleural effusion occurred at the same time. The patients were treated with CAD regimen (DXM $40 \mathrm{mg}$ d1-4+CTX $0.6 \mathrm{~g}$ $\mathrm{d} 1-3+$ Epirubicin $60 \mathrm{mg} \mathrm{d} 4)$. The related images and blood tests showed that the patients were not in remission.

At this time, the patient experienced a total of 8 cycles BEACOPP, 3 cycles IGDV Hodgkin regimen, 4 cycles BV,
Allo-HSCT, multiple local radiotherapy and 1 cycle of CAD, all failed to achieve long-term remission, which can be classified as R/R HL which is ineffective to radiotherapy and chemotherapy and Allo-HSCT. This led us to further search for more effective solutions.

Referring to the literature and guidelines, immune checkpoint therapy has brought a gratifying effect in the treatment of hematological tumors, especially HL [3], which provides a new idea for our treatment. The results of pharmacokinetic analysis and clinical observation showed that low-dose PD-1 inhibitor was potentially effective in the treatment of solid tumors [4]. In additionally, it has been reported that R/R HL patients who have previously received Allo-HSCT or not, have significant responses to low-dose of nivolumab and pembrolizumab [5], so we chose the minimum dose of PD-1 inhibitor $40 \mathrm{mg}$ for immunotherapy. The patients showed transient facial and retroauricular skin hyperemia and without other adverse reactions. We gave the patients a total of 12 low-dose PD-1 inhibitors (40 mg/14days), and several times of chest CT evaluation showed that the primary mass was smaller than before, and the nodules scattered in both lungs were reduced. Remission lasted for 7 months.

During the 13th PD-1 inhibitor treatment, imaging showed that the lung and mediastinal lesions were enlarged than before, indicating disease's activity. Increase the dose of PD-1 inhibitors, the patient received three times of immunotherapy with $80 \mathrm{mg}$ PD-1 inhibitors and two cycles of chemotherapy + targeted therapy (gemcitabine $1000 \mathrm{mg} / \mathrm{m}^{2} \mathrm{~d} 1+$ Vindesine sulfate $3 \mathrm{mg} / \mathrm{m}^{2} \mathrm{~d} 1+15 \mathrm{mg} / \mathrm{m}^{2} \mathrm{~d} 1+\mathrm{BV} 150 \mathrm{mg} \mathrm{d} 8$ ). Since then, the patient's condition is stable. Combined with previous treatment regimens, the patient is sensitive to $\mathrm{BV}$ treatment. So we have given four courses of BV $(100 \mathrm{mg})$ to consolidation therapy, and after the third course of treatment, the patient achieved partial remission. However, progress during treatment showed that patients were no longer sensitive to $\mathrm{BV}$.

According to the manual, we gradually adjusted the PD-1 dose. The final dose was increased to the manual recommended standard dose of $3 \mathrm{mg} / \mathrm{kg}$, and the patient received remission. Up to now, the patient has been in the state of remission for a long time under the maintenance therapy of $3 \mathrm{mg} / \mathrm{Kg}$ PD-1 inhibitor.

\section{Discussion}

HL is a common hematological malignant tumor, which usually occurs in young and middle-aged people [6]. According to the NCCN guidelines, the common treatment schemes are ABVD, BEACOPP, StandfordV and so on [3]. Most patients will be in complete remission after initial treatment and achieve long-term disease control. However, there are still a small number of patients with recurrent attacks, and the progression of the disease eventually leads to death. This paper reports a case of R/R HL that relapses within a short period of time after transplantation.

After being diagnosed with HL at the age of 18, the patient experienced a variety of treatments, including bone marrow 
transplantation, but all failed to achieve long-term remission. Then we tried immunotherapy including BV and PD-1 inhibitors. Despite progress during treatment, the patient eventually achieved long-term remission with the maintenance of standard doses of PD-1 inhibitors.

The traditional chemotherapy regimen does not get the ideal therapeutic effect on patient, but the rapid development of immunotherapy in recent years has brought new hope for patient. BV is a kind of antibody-drug coupling, which recognizes and binds tumor cells which expressing CD30+ through the combination of monoclonal antibodies and small molecular antineoplastic drugs, and then make chemical small molecular drugs enters tumor cells through endocytosis of cells $[7,8]$. It can be used in combination with other chemotherapeutic drugs or alone in recurrent lymphoma after transplantation, it showed a good complete remission rate in II/III clinical trials [9]. The patient we reported received four cycles of BV before transplantation and responded well, so we treated the patient again in the event of recurrence after transplantation, but did not achieve the desired effect like past.

The above enlightens us to explore the reasons why follow-up BV treatment is ineffective. We often think that antigen down-regulation is a potential mechanism of drug resistance to antigen therapy. However, the resistance to BV in HL is not due to the decreased expression of CD30, but to the up-regulation of multiple drug resistance gene MDR1 and the emergence of cytotoxic drug monomethyl auristatin E (MMAE) resistance [10]. It was found that in the mouse model, the effect of $\mathrm{BV}$ on $\mathrm{HL}$ was restored after competitively inhibiting the expression of MDR1 [11]. Phase I trials that combined with $\mathrm{BV}$ and CsA were performed in $86 \%$ of patients with $\mathrm{R} / \mathrm{R}$ HL insensitive to $\mathrm{BV}$ [11]. The results showed that the rates of ORR and CR were $75 \%$ and $42 \%$, respectively [11]. However, the subsequent adverse reactions, such as myelosuppression, also greatly limit the clinical use. It is suggested that the simultaneous detection of the expression level of CD30 may play a better role in guiding the use of drugs when using BV.

PD-1 is the key immunosuppressive receptor for T cells to activate the second signal [12]. PD-1 inhibitors activate $T$ cells to fight tumor by antagonizing the binding of PD-1 to the specific receptor PD-L1, and obtain a good response in solid tumors [12]. It has a good effect in hematological tumors, especially in patients with HL [13]. The role of PD-1 inhibitors as consolidation therapy in patients with Allo-HSCT is still controversial. As we all know, the GVL effect after transplantation is an important anti-tumor step, and the loss of GVL effect is considered to be one of the main causes of recurrence of metastatic diseases, which is also the most common cause of death and treatment failure after Allo-HSCT [14]. As early as in the study of leukemia, it has been found that recurrence after Allo-HSCT is related to the killing of tumor cells by PD-1-mediated loss of donor T cells, after down-regulating the expression of PD-1, it may restore the effect of GVL on recurrent AML [15]. Obviously, blocking the inhibitory effect of PD-1 on immune cells may be beneficial to patients after Allo-HSCT. However, as an immune checkpoint, PD-1/PD-L1 has not only disadvantages. PD-1/PD-L1 can significantly regulate the GVHD effect, and the interaction between PD-1 and PD-L1 can inhibit the graft-versus-host immune response mediated by Th1 / Th17 $[16,17]$. Therefore, how to balance the advantages and disadvantages of PD-1/PD-L1 blocking is a problem that we continue to pay close attention to. Both preclinical and clinical data suggest that the shorter interval between Allo-HCT and ICI treatment is associated with a higher risk of immune complexity [17]. We did not use PD-1 inhibitors for the first time, but 9 months after transplantation, which may be one of the reasons why the patients did not show serious adverse reactions.

At present, the recommended dose of lymphoma PD-1 inhibitor is $3 \mathrm{mg} / \mathrm{Kg}$ or $240 \mathrm{mg}$ fixed dose. However, the results of pharmacokinetic analysis and clinical observation show that low dose PD-1 especially monoclonal antibody has potential curative effect on solid tumor $[4,18]$. In additionally, it has been reported that R/R HL patients who have previously received Allo-HSCT or not, have significant responses to low dose nivolumab and pembrolizumab $[5,19]$. To be on the safe side, the low-dose PD-1 inhibitor (40 mg) we used at first. After that, patients not only got a good response, but also without show serious adverse reactions, which provided a guarantee for gradually increasing the therapeutic dose of PD-1 inhibitor after the later progression of the disease. However, the patient inevitably progressed, until the dose of the PD-1 inhibitor was gradually increased to the recommended dose of $3 \mathrm{mg} / \mathrm{kg}$ in the instructions, the disease was controlled. Since then, the patient has been treated with PD-1 inhibitor and is still in the state of remission.

It has been found that, nivolumab (standard dose $240 \mathrm{mg}$ ) sequential N-AVD regimen has good efficacy and safety in newly diagnosed III-IV patients with advanced $\mathrm{cHL}$ and $\mathrm{cHL}$ adults with large fast masses or extranodal diseases [20]. It makes us wonder whether the initial use of large doses of PD-1 inhibitors can achieve early remission. Besides, although the current guidelines recommend that patients with high risk factors have little chance of recurrence after 1-2 years of maintenance therapy with PD-1 inhibitors [2, 3], for this patient, who has experienced early recurrence and poor response to many chemotherapeutic drugs and radiotherapy, even relapse after transplantation and $\mathrm{BV}$ resistance. The duration of PD-1 inhibitor maintenance treatment, how to prevent PD-1 inhibitor treatment resistance and select subsequently treatment options after drug resistance? Above, all is worth us to continue to think and explore the problem.

\section{Conclusion}

For patients with Hodgkin's lymphoma who have undergone multiple conventional chemotherapy regimens but have not achieved desired therapeutic outcomes, the rapid development of immunotherapy has brought new hope to patients. However, for such patients with recurrence and progression of disease after transplantation, the dosage, timing and maintenance time of immunosuppressive drugs 
are still questions that we continue to study and explore.

\section{Statement}

The manuscript has been read and approved by all the authors.

\section{Conflict of Interest}

All the authors do not have any possible conflicts of interest.

\section{References}

[1] Press OW, Li H, Schöder H, Straus DJ, Moskowitz CH, LeBlanc M, Rimsza LM, Bartlett NL, Evens AM, Mittra ES, LaCasce AS, Sweetenham JW, Barr PM, Fanale MA, Knopp MV, Noy A, Hsi ED, Cook JR, Lechowicz MJ, Gascoyne RD, Leonard JP, Kahl BS, Cheson BD, Fisher RI, Friedberg JW: US Intergroup Trial of Response-Adapted Therapy for Stage III to IV Hodgkin Lymphoma Using Early Interim Fluorodeoxyglucose-Positron Emission Tomography Imaging: Southwest Oncology Group S0816. J Clin Oncol. 2016, 34: 2020-2027. 10.1200/jco.2015.63.1119.

[2] Ansell SM: Hodgkin lymphoma: 2018 update on diagnosis, risk-stratification, and management. Am J Hematol. 2018, 93: 704-715. 10.1002/ajh.25071.

[3] Hoppe RT, Advani RH, Ai WZ, Ambinder RF, Armand P, Bello CM, Benitez CM, Bierman PJ, Boughan KM, Dabaja B, Gordon LI, Hernandez-Ilizaliturri FJ, Herrera AF, Hochberg EP, Huang J, Johnston PB, Kaminski MS, Kenkre VP, Khan N, Lynch RC, Maddocks K, McConathy J, McKinney M, Metzger M, Morgan D, Mulroney C, Rabinovitch R, Rosenspire KC, Seropian S, Tao R, Winter JN, Yahalom J, Burns JL, Ogba N: Hodgkin Lymphoma, Version 2.2020, NCCN Clinical Practice Guidelines in Oncology. 2020, 18: 755. 10.6004/jncen.2020.0026.

[4] Stroh M, Winter H, Marchand M, Claret L, Eppler S, Ruppel J, Abidoye O, Teng SL, Lin WT, Dayog S, Bruno R, Jin J, Girish S: Clinical Pharmacokinetics and Pharmacodynamics of Atezolizumab in Metastatic Urothelial Carcinoma. Clin Pharmacol Ther. 2017, 102: 305-312. 10.1002/cpt.587.

[5] Lepik KV, Fedorova LV, Kondakova EV, Zalyalov YR, Babenko EV, Lepik EE, Kotselyabina PV, Beynarovich AV, Popova MO, Volkov NP, Stelmakh LV, Baykov VV, Moiseev IS, Mikhailova NB, Kulagin AD, Afanasyev BV: A Phase 2 Study of Nivolumab Using a Fixed Dose of $40 \mathrm{mg}$ (Nivo40) in Patients With Relapsed/Refractory Hodgkin Lymphoma. Hemasphere. 2020, 4: e480. 10.1097/HS9.0000000000000480.

[6] Punnett A, Tsang RW, Hodgson DC: Hodgkin lymphoma across the age spectrum: epidemiology, therapy, and late effects. Seminars in radiation oncology. 2010, 20: 30-44. 10.1016/j.semradonc.2009.09.006.

[7] Francisco JA, Cerveny CG, Meyer DL, Mixan BJ, Klussman K, Chace DF, Rejniak SX, Gordon KA, DeBlanc R, Toki BE, Law CL, Doronina SO, Siegall CB, Senter PD, Wahl AF: cAC10-vcMMAE, an anti-CD30-monomethyl auristatin E conjugate with potent and selective antitumor activity. Blood. 2003, 102: 1458-1465. 10.1182/blood-2003-01-0039.
[8] Doronina SO, Toki BE, Torgov MY, Mendelsohn BA, Cerveny CG, Chace DF, DeBlanc RL, Gearing RP, Bovee TD, Siegall CB, Francisco JA, Wahl AF, Meyer DL, Senter PD: Development of potent monoclonal antibody auristatin conjugates for cancer therapy. Nat Biotechnol. 2003, 21: 778-784. 10.1038/nbt832.

[9] Younes A, Gopal AK, Smith SE, Ansell SM, Rosenblatt JD, Savage KJ, Ramchandren R, Bartlett NL, Cheson BD, de Vos S, Forero-Torres A, Moskowitz CH, Connors JM, Engert A, Larsen EK, Kennedy DA, Sievers EL, Chen R: Results of a pivotal phase II study of brentuximab vedotin for patients with relapsed or refractory Hodgkin's lymphoma. J Clin Oncol. 2012, 30: 2183-2189. 10.1200/jco.2011.38.0410.

[10] Chen R, Hou J, Newman E, Kim Y, Donohue C, Liu X, Thomas SH, Forman SJ, Kane SE: CD30 Downregulation, MMAE Resistance, and MDR1 Upregulation Are All Associated with Resistance to Brentuximab Vedotin. Mol Cancer Ther. 2015, 14: 1376-1384. 10.1158/1535-7163.Mct-15-0036.

[11] Chen R, Herrera AF, Hou J, Chen L, Wu J, Guo Y, Synold TW, Ngo VN, Puverel S, Mei M, Popplewell L, Yi S, Song JY, Tao S, Wu X, Chan WC, Forman SJ, Kwak LW, Rosen ST, Newman EM: Inhibition of MDR1 Overcomes Resistance to Brentuximab Vedotin in Hodgkin Lymphoma. Clin Cancer Res. 2020, 26: 1034-1044. 10.1158/1078-0432.Ccr-19-1768.

[12] Hui E, Cheung J, Zhu J, Su X, Taylor MJ, Wallweber HA, Sasmal DK, Huang J, Kim JM, Mellman I, Vale RD: T cell costimulatory receptor $\mathrm{CD} 28$ is a primary target for PD-1-mediated inhibition. Science. 2017, 355: 1428-1433. 10.1126/science.aaf1292.

[13] Bröckelmann PJ, Goergen H, Keller U, Meissner J, Ordemann R, Halbsguth TV, Sasse S, Sökler M, Kerkhoff A, Mathas S, Hüttmann A, Bormann M, Zimmermann A, Mettler J, Fuchs M, von Tresckow B, Baues C, Rosenwald A, Klapper W, Kobe C, Borchmann P, Engert A: Efficacy of Nivolumab and AVD in Early-Stage Unfavorable Classic Hodgkin Lymphoma: The Randomized Phase 2 German Hodgkin Study Group NIVAHL Trial. JAMA oncology. 2020, 6: $872-880$. 10.1001/jamaoncol.2020.0750.

[14] Negrin RS: Graft-versus-host disease versus graft-versus-leukemia. Hematology American Society of Hematology Education Program. 2015, 2015: 225-230. 10.1182/asheducation-2015.1.225.

[15] Asakura S, Hashimoto D, Takashima S, Sugiyama H, Maeda Y, Akashi K, Tanimoto M, Teshima T: Alloantigen expression on non-hematopoietic cells reduces graft-versus-leukemia effects in mice. J Clin Invest. 2010, 120: 2370-2378. $10.1172 / \mathrm{JCI} 39165$

[16] Norde WJ, Maas F, Hobo W, Korman A, Quigley M, Kester MG, Hebeda K, Falkenburg JH, Schaap N, de Witte TM, van der Voort R, Dolstra H: PD-1/PD-L1 interactions contribute to functional T-cell impairment in patients who relapse with cancer after allogeneic stem cell transplantation. Cancer Res. 2011, 71: 5111-5122. 10.1158/0008-5472.CAN-11-0108.

[17] Saha A, Aoyama K, Taylor PA, Koehn BH, Veenstra RG, Panoskaltsis-Mortari A, Munn DH, Murphy WJ, Azuma M, Yagita H, Fife BT, Sayegh MH, Najafian N, Socie G, Ahmed R, Freeman GJ, Sharpe AH, Blazar BR: Host programmed death ligand 1 is dominant over programmed death ligand 2 expression in regulating graft-versus-host disease lethality. Blood. 2013, 3062-3073. 10.1182/blood-2013-05-500801. 
[18] Yoo SH, Keam B, Kim M, Kim SH, Kim YJ, Kim TM, Kim DW, Lee JS, Heo DS: Low-dose nivolumab can be effective in non-small cell lung cancer: alternative option for financial toxicity. ESMO Open. 2018, 3: e000332. 10.1136/esmoopen-2018-000332.

[19] Hwang YY, Khong PL, Kwong YL: Low-dose nivolumab induced remission in refractory classical Hodgkin lymphoma. Ann Hematol. 2017, 96: 1219-1220. 10.1007/s00277-017-3007-9.
[20] Ramchandren R, Domingo-Domènech E, Rueda A, Trněný M, Feldman TA, Lee HJ, Provencio M, Sillaber C, Cohen JB, Savage KJ, WillenbacherR/R W, Ligon AH, Ouyang J, Redd R, Rodig SJ, Shipp MA, Sacchi M, Sumbul A, Armand P, Ansell SM: Nivolumab for Newly Diagnosed Advanced-Stage Classic Hodgkin Lymphoma: Safety and Efficacy in the Phase II CheckMate 205 Study. J Clin Oncol. 2019, 37: 1997-2007. 10.1200/jco.19.00315. 